\title{
SPATIAL CHARACTERIZATION OF TELECOMMUNICATION SATELLITES VISIBLE ABOVE THE REPUBLIC OF SERBIA
}

\author{
SLAVIŠA ĐUKANOVIĆ ${ }^{1}$ ， VLADIMIR MLADENOVIĆ ${ }^{* *}$ MILAN GLIGORIJEVIĆ ${ }^{1}$, \\ DANIJELA MILOŠEVIĆ ${ }^{2}$, IVONA RADOJEVIĆ ALEKSIĆ
}

${ }^{1}$ University of Criminal Investigation and Police Studies, Belgrade, Serbia

${ }^{2}$ Faculty of Technical Sciences in Cacak, University of Kragujevac, Cacak, Serbia

\begin{abstract}
This paper deals with the general technical, spatial, and temporal characteristics of satellite telecommunications systems. Particular attention was paid to the peculiarities of the territory of the Republic of Serbia in terms of implementation and use of modern satellite telecommunication infrastructure. The overview shows how and on which way to use various satellite telecommunication systems to configure and exploit next-generation networks, especially modern communications such as $5 \mathrm{G}$ technology and IoT. Their work cannot be imagined without the high speeds and high frequencies that allow us to transmit a wealth of information - from short messages/news to HD video on a mobile phone. We need either a base station network or in the case of the high seas or areas where the base station system is difficult to imagine, we need satellite communication. The paper presents data that give a numerical and graphical overview of geostationary satellites visible from the territory of the city of Belgrade, depending on the orbital position and the associated angle of azimuth and elevation, which would also be valid for the leading territory of Serbia.
\end{abstract}

Keywords: Satellite's orbites, 5 G technologies, IoT, Azimuth, Elevation.

In general, the reconnaissance of satellite systems

\section{INTRODUCTION}

Satellite telecommunication systems represent a significant part of the global telecommunication market. Alongside the evolution of technology, these systems, whether global or local, provide users with a wide range of services. The constant application of commercial satellite systems in military, diplomatic, and industrial purposes arises the question of how to protect signals thus transmitted. The reconnaissance of telecommunications is a complex activity that implies a wide range of actions: the examination of radio-frequency spectrum, signal reception, goniometric assessment, technical analysis of signals, and data processing. The complexity of the telecommunication reconnaissance process is directly associated with compound communication transmission techniques applied. Thus, new possibilities in the implementation of various detection and signal and parameter estimation methods emerge aimed at quality reconnaissance of telecommunication via specified systems.

The main function of the reconnaissance system is to detect the object significant for observation and to continuously monitor it regardless of the time variability of its characteristics, the presence of the distraction signals, and the influence of present whirs. The application of the time-varying system parameters estimation increases the effectiveness and robustness of the reconnaissance systems.

\footnotetext{
* Corresponding author: vladimir.mladenovic@ftn.kg.ac.rs MATHEMATICS, COMPUTER SCIENCE AND MECHANICS represents one of the crucial components of the electronic reconnaissance as a whole. The reconnaissance of satellite systems provides technical data (technical characteristics of satellite systems in general, satellite telecommunication systems and signals used in satellite telecommunications) and operativetechnical data (levels of operational and technical application, the type and purpose of the satellite, satellite radio-transmission intensity data, etc.).

The possibility of detecting and monitoring a satellite, in general, is considered through the implementation of radar reconnaissance techniques, radio-reconnaissance, IC reconnaissance, and using laser systems. The term reconnaissance implies the analyzed possibility to discern the observed object as a satellite and determine that the examined signal originates from the satellite, not any other source in the same spacial sector.

The methodology of the satellite telecommunication reconnaissance is divided into several phases:

- The detection, observation and monitoring phase with the application of the estimation techniques,

- The identification phase and the analysis phase.

The detection, observation, and monitoring phase implies space exploration, tracking mobile satellites via antenna systems of radio-reconnaissance station. These are followed by the application of characteristic parameters estimation methods and techniques, radio spectrum examination, representation, detection, and primary analysis of the signals. The identification phase comprises the elements of the spatial and frequency satellite categorization, primary function identification, and a 
confirmation that a single satellite originates from a satellite system. The analysis procedure includes following activities: analyzing the satellite orbit parameters, satellite frequency agility analysis, determination of type and transmission parameters (modulation), determination of the correspondents with which the connection is maintained, issuing command and synchronization impulses, extraction of the signals carrying a useful message and analytic processing of received messages.

The main goal of this paper is the representation of the territorial peculiarities of the Republic of Serbia in terms of implementation and use of modern satellite telecommunication infrastructure. The paper points out the way to use various satellite telecommunication systems to configure and exploit next-generation networks, especially modern communications such as $5 \mathrm{G}$ technology and IoT.

\section{CLASSIFICATION OF SATELLITES BASED ON TYPE OF ORBIT}

Communications satellites can be classified by the service they perform. However, these satellites are classified into those for radio-diffusion, mobile, and fixed satellite services. Communications satellites that provide mobile satellite services are used for Mobile Satellite Communications - MSC in applications, both rural and urban radio systems. Satellites can be classified by the orbital height of a satellite. Thus, CCIR distinguishes four main types of satellites:

- Low-Earth Orbit Satellites circling at altitudes from 100 to $5000 \mathrm{~km}$ above the Earth with orbital periods from 2 to 4 hours.

- Medium-Earth Orbit Satellites circling at altitudes from 5000 to $20000 \mathrm{~km}$ above the Earth with orbital periods from 4 to 20 hours.

- High-Earth Orbit Satellites circling at altitudes about $36000 \mathrm{~km}$ above the Earth with orbital periods of 24 hours.

- Orbits at great altitudes, about $36000 \mathrm{~km}$ above the Earth (Gligorijević et al., 2011).

The following satellites can be distinguished depending on the orbit and the satellite's relative circling above the Earth. Synchronous satellites have an average period of stellar rotation around the body equal to the average rotational period of the body on its axis, thus they can be referred to as geosynchronous.

If these satellites orbit on the equatorial plane, they are referred to as - geostationary satellites. Their subsatellite point occupies an unvaried position, so to ground observers it appears motionless, i.e. stationary. Since these periods last about $23 \mathrm{~h}$ and 56 minutes, these satellites circle the Earth in a sidereal day. Their orbit plane may have null or any other inclination towards the Earth's equatorial plane, such as polar or satellites with other inclination.

Subsynchronous satellites have the average rotational period of the body orbited on its axis equal to the entire multiple of the average rotational period of the satellite around the body. It means that they orbit over the same spot on Earth twice, or they circle it twice, i.e. their orbit lasts 12 hours. Elliptical orbit satellites and other satellites with circular orbit inclination belong to this category.

Non-synchronous satellites circle the earth more than twice in 24 hours, i.e. their orbit period is longer than 12 hours.

Satellites may be classified by the type of activity regarding the territory of the Republic of Serbia (Andrews \& Phillips, 1998):

1) Satellites that do not broadcast signals in the direction of the Republic of Serbia (spare, malfunctioning or remnants of the satellites, satellites out of work, observatory satellites which emit the results of their observation only above certain spots outside the territory of the Republic of Serbia, satellites that are activated only in emergencies - crises and wars)

2) Satellites that probably broadcast signals in the direction of the territory of the Republic of Serbia. It is difficult to detect what these satellites transmit (telecommunication relay satellites with strongly directed spot beam radio transmissions, satellites that broadcast radio signals through widened spectrum and satellites which broadcast for a short period);

3) Satellites that publicly broadcast in the direction of the territory of the Republic of Serbia (satellites with civil purposes: telecommunication satellites, radio-diffusion, meteorological, navigation, satellites for transmitting the precise time signal; military satellites and other satellites of classificatory purposes: navigation, meteorological, observation, research, diplomatic, etc.)

When identifying satellites and their activity regarding the territory of the Republic of Serbia, movement parameters and types of satellite orbits are especially important. These are:

- Orbit shape with data on perigee, apogee, and inclination (circular and elliptic),

- Height and type of orbit, and

- Speed, that is a period of circulating the Earth.

Orbit's height and shape, satellite's speed and direction, and effective reflection area of the satellite are significant parameters during radar characterization (Panić et al., 2013).

Classification of the satellites regarding parameters significant for radio-characterization is possible to determine considering frequency spectrum and working frequencies, types of transmission, modulation types, and shapes of radiation diagram (spot beam, global beam, and multi-beam).

\section{Types of satellite orbits}

Taking into consideration the fact that astronomical objects freely move and mutually attract in the interplanetary space following the well-known laws, it is impossible to construct a satellite that would be fixed because it would be domed. 
Having this in mind, artificial satellites can orbit in the circular and elliptical orbit planes of the geocentric coordinate system. Apart from ESO (Elliptical Satellite Orbit), there are three other circular orbits: GSO (Geostationary Satellite Orbit), PSO (Polar satellite Orbit), and inclined circular geosynchronous orbit, figure 1.

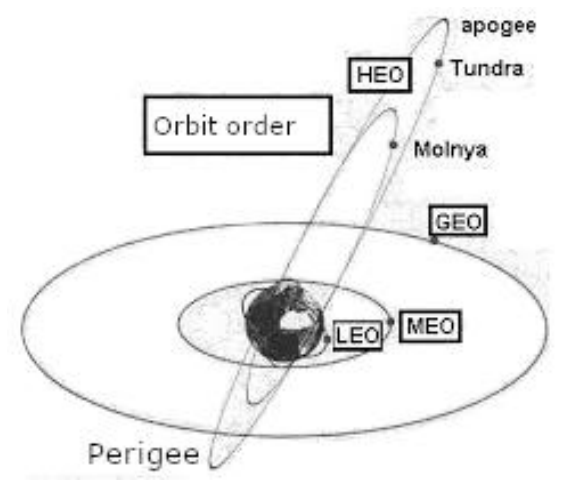

Figure 1. Overview of satellite orbit types.

Satellites circulation in the Earth's orbit can be depicted using Newton's Law of Gravitation while the concept of the elliptical or circular orbit is based on three Kepler's Laws. After launching, a satellite remains in the orbit due to balance established between the first force or the Earth's gravitational centripetal attraction and the second force, i.e. satellite's centrifugal acceleration in the circular orbit. Other forces have a negligible impact on the satellite's orbit.

\section{Elliptical orbit}

An elliptical orbit is an ellipse with the inclination angle of about $63^{\circ}$ and an orbit period of 12 hours. It belongs to a group of subsynchronous orbits. Satellites in elliptical orbits cover polar zones and wider zones of the globe with the transition time from 8 to 10 hours out of 12 hours. The satellite's velocity varies. It is approximately about $10150 \mathrm{~m} / \mathrm{s}$ at perigee and about $1610 \mathrm{~m} / \mathrm{s}$ at apogee, while the velocity of the point on the equator is about $465 \mathrm{~m} / \mathrm{s}$. Marking the traces of the elliptical path is a far more complicated process due to inconsistencies that appeared as a result of the complex interrelation between satellite's speed and the Earth's rotation.

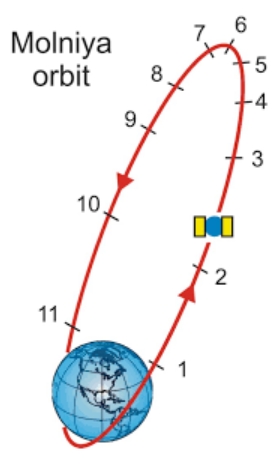

Figure 2. Elliptic satellite orbit.
Russian EOS MOLNIA-1 is a prototype of satellites in this orbit, Figure 2 (David 2020).

General data of the Molnya satellite are given in Table 1.

Table 1. Basic information about the MOLNIA satellite.

\begin{tabular}{|l|l|}
\hline EOS Parameters & Technical characteristics \\
\hline Apogee height & $39957 \mathrm{~km}$ (above north hemisphere) \\
\hline Perigee height & $548 \mathrm{~km}$ (above south hemisphere) \\
\hline Inclination & $63.4^{\circ}$ \\
\hline Eccentricity & $0.74^{\circ}$ \\
\hline Argument of perigee & $270^{\circ}$ \\
\hline Approximate period & 12 hours \\
\hline
\end{tabular}

Disadvantages of the EOS are due to a relative satellite orbiting in relation to the Earth and the fact that ZSS must be equipped with a highly complicated antenna system for reconnaissance of the satellites, though this could be achieved by automatic search. With EOS, the period of satellite obscuration should be taken into consideration to avoid energy loss.

The fact that less energy is needed to launch the EOS and that no additional motors for orbit correction are necessary are the advantages of the EOS. Thus energetic possibilities that EOS electronic devices possess are significantly greater than ones GSS has. Besides, another advantage of EOS satellites is the fact that ZSS in geographic positions at high latitudes there are greater elevation angles that enable loss reduction during propagation and decrease in satellite line blocking.

\section{Circular satellite orbit}

There are three different types of circular satellite orbits depending on the angle of inclination formed between the satellite's orbital plane and the equatorial plane. These are: polar when $i=90^{\circ}$ (orbit 1 ), inclined with the proportion $0^{\circ}<i<90^{\circ} \mathrm{i}$ $90^{\circ}<i<180^{\circ}$ (orbit 2 ), and equatorial GSS when $i=0^{\circ}$ (orbit 3 ).

\section{Geostationary (circum equatorial) orbit}

If an artificial satellite is orbiting in the equatorial plane from west to east following the direction of Earth's rotation, its angular velocity will match the angular velocity of the Earth's rotation on its axis, thus to a ground observer, the satellite appears motionless. We are referring to synchronous GSS which presents a constellation of the INMARSAT system, (Đukanović 2006a). The GSS subsatellite points occupy various positions on the equator depend on their number and arrangement, while the length of equatorial arc from where an artificial satellite can be seen is $162^{\circ} 36^{\prime} 30^{\prime \prime}$ or $18102 \mathrm{~km}$. An equatorial arc length, which is not covered by two GSS, is $34^{\circ} 47^{\prime}(3872 \mathrm{~km})$, while the total length of the equatorial arc from where it is possible to see two or three artificial satellites is $14232 \mathrm{~km}(26.21 \%)$, table 2. 
Table 2. Parameters of GSO.

\begin{tabular}{|l|l|}
\hline GSS parameters & Technical characteristics \\
\hline Height above the equator & $35784 \mathrm{~km}$ \\
\hline $\begin{array}{l}\text { Periods of rotation relative to the } \\
\text { Sun. }\end{array}$ & 24 hours \\
\hline BY. in relation to the sidereal day & $23 \mathrm{~h} 56 \mathrm{~min}$ i $4.091 \mathrm{sec}$ \\
\hline Circulation speed & $3076 \mathrm{~m} / \mathrm{sec}$ \\
\hline Orbital inclination & $0^{\circ}$ \\
\hline Earth's surface coverage & $42.5 \%\left(0^{\circ}\right.$ elevacije $)$ \\
\hline Global coverage & $3 \mathrm{GSS}$ with shift of $120^{\circ}$ \\
\hline Theoretical coverage of the area & $<81^{\circ} \mathrm{N} \& \mathrm{~S}$ \\
\hline
\end{tabular}

The advantages of GSO are simple retrieval, there are no problems with the transmission, because the losses during signal propagation are quite limited, except during weather variations, almost constant connection range. Only one GSS is sufficient for regional uninterrupted coverage, high antenna signal amplification.

The disadvantages of GSO are the delay due to propagation, loss of free space, uncovered polar regions, and very important for MSC, there is no possibility of direct connections of MSS in one, with ZSS in another ocean area.

\section{Circuminclination orbit}

The circumclination orbit is obtained when the plane of the satellite's orbit is inclined at a certain angle to the equatorial plane, which is called the inclination angle. It can be defined as the angle that coincides between the equator and the sub-satellite point when the satellite enters the northern hemisphere and can have values from $0^{\circ}$ to $90^{\circ}$ and from $90^{\circ}$ to $180^{\circ}$. In the case of a synchronous orbit, the satellite will not be visible from the Earth in a constantly determining direction but will be visible from the same point of the Earth's ball at different times and at different heights above the horizon. Thus, in this way, a trace of the path of subsatellite points in the shape of an elongated figure eight can be projected, the shape of which depends on the size of the inclination angle.

There are two circumminclination orbits that can be used for all forms of mobile applications.

\section{CIRCULAR INCLINATION ORBIT}

This orbit is used for the existing global navigation system, whose configuration consists of 24 satellites in three equilateral orbits. At a minimum, three satellites in each of the three orbits are equipped with polar communication equipment, to ensure continuous coverage of the polar zones (Đukanović 2006b).

\section{Circumpolar orbit}

When the inclination of the plane of the satellite's path towards the equator is $90^{\circ}$, a circumpolar orbit is obtained, which is in the plane of the Earth's axis. If the satellite orbit time is synchronous, a synchronous circular path is obtained. The relative position of such a satellite changes constantly, and every 24 hours it appears at the zenith of the same point on Earth, whose trace has the shape of a broad figure eight. However, for the case of a 12-hour orbit, the trajectory has the shape of two cross eights, and for the case of a 6-hour orbit, the trail is in the shape of four elongated eights. This type of satellite enables better coverage of regions at the highest latitudes and polar regions above $70^{\circ} \mathrm{N}$ and $\mathrm{S}$, which are poorly or not at all covered by the existing GSS constellation.

\section{Geometric relations of satellite orbit}

During the design and operation of MSK, it is necessary to know the launch of satellites and orbital elements, all parameters, and laws of satellite shortening, as well as geographical and horizontal coordinates of satellites and their mutual relations. Kepler's laws describe the behavior of natural satellites if the mass of the central body is so far away that it is considered concentrated in its center and when their orbits are not under the influence of some other systems.

However, these conditions are not fully met when it comes to the Earth and its artificial satellites, since their mutual distances are not great, because the Earth is flattened and the masses in its interior are not properly distributed. Therefore, the satellites move unevenly, ie faster or slower on certain sections of the orbit, and this represents a certain deviation from the essence of Kepler's laws (Đukanović 2004).

The characteristics of an elliptical orbit are determined from the elements of the ellipse and its position relative to the Earth, Figure 3. Ellipse parameters, such as eccentricity e, major semiaxis $a$, minor semiaxis $b$, the axis between the center of the Earth and ellipse $c$, and focal parameter $p$, are obtained from the following expressions. The equation of the ellipse (Kepler trajectory) from the polar coordinates has the form:

$$
r=\frac{p}{1+e \cos \theta}
$$

where the radius of the orbit is $r=R+H$, while $\mathrm{e}$ is a right anomaly, and $\theta$ is the angle between the directions from the center of the Earth to the perigee and the satellite, and $E$ is an eccentric anomaly, whose expressions are:

$$
\cos E=\frac{\cos \theta+e}{1+e \cos \theta}
$$

The speed of the satellite at point $S$, distant from the center of the Earth by $r$, is obtained from the expression:

$$
v=\sqrt{\mu\left(\frac{2}{r}-\frac{1}{a}\right)}
$$

where $\mu$ is the gravitational constant $\left(\times 10^{5} \mathrm{~km}^{3} / \mathrm{s}^{2}\right)$, or Kepler's constant, the major semiaxis $\alpha$. 


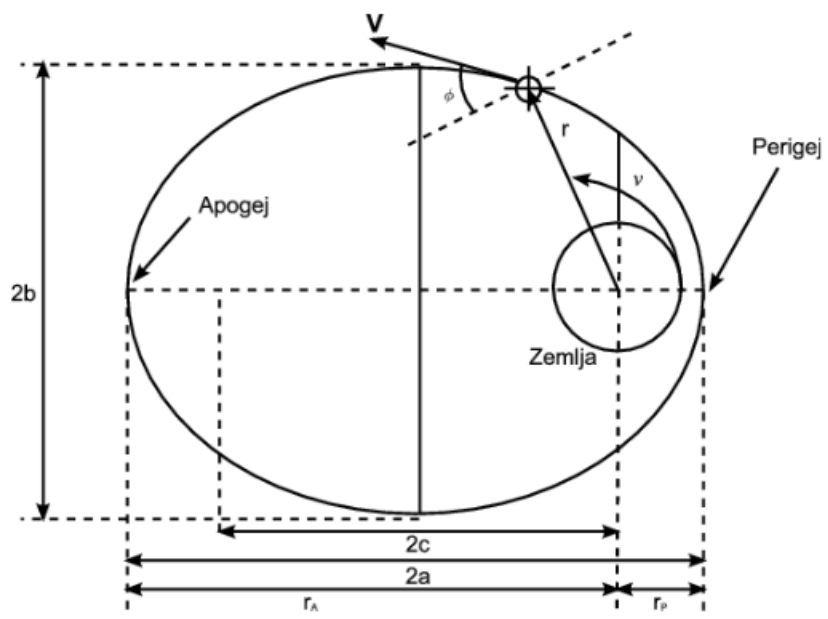

Figure 3. Elliptical satellite orbit parameters.

The behavior of artificial Earth satellites from the moment they enter orbit, similar to natural satellites, is determined by the laws of celestial mechanics. Thus, according to Kepler's third law, the sidereal or stellar time of a satellite orbit around the Earth in an elliptical orbit is:

$$
t=2 \pi \sqrt{\frac{a^{3}}{\mu}}
$$

where $R$ is the mean radius of the Earth, while $h$ is the height of the orbit.

A circular orbit is a special case of an elliptical orbit that arises from the following relations: $a=b=r$ and $e=0$. The speed of the satellite in a circular orbit can be determined from the following expression:

$$
v=\sqrt{\frac{\mu}{r}}=631.65 \sqrt{R+h}
$$

The duration of one full rotation of the Earth in relation to the standing stars is $T=23 \mathrm{~h} 56 \mathrm{~min} 4.09 \mathrm{~s}$. If the satellite rotates around the Earth in the direction of rotation, the apparent rotation time $t^{\prime}$ will be:

$$
t^{\prime}=\frac{T \cdot t}{T-t}
$$

The satellite is geostationary if $t=T$. Synodic time is obtained between successive passages of satellites above the meridian, whose expression reads:

$$
T=\frac{t}{[1-\Omega t / 2 \pi]}
$$

The synodic day is the time interval between two consecutive passages of the solar center through the meridian of the same name.

The Table 3 shows that a geostationary orbit can be established at an altitude of about $36000 \mathrm{~km}$. The height of the satellite's orbit can be calculated based on the expression:

$$
\begin{aligned}
& H=(r-6378) \\
& H=\sqrt[3]{\frac{\mu t^{2}}{4 \pi^{2}}}-R
\end{aligned}
$$

Table 3. Overview of dependence, time of orbiting the earth on the height of the orbiting satellite.

\begin{tabular}{|c|c|c|}
\hline Orbital height in $\mathrm{km}$ & $t(\mathrm{~h})$ & $T(\mathrm{~h})$ \\
\hline 1700 & 2 & 2.18 \\
\hline 10400 & 6 & 8 \\
\hline 14000 & 8 & 12 \\
\hline 20200 & 12 & 24 \\
\hline 36000 & 24 & - \\
\hline
\end{tabular}

It can be seen from expression (6) and Table 4 that the speed of a satellite does not depend on its mass but decreases with increasing altitude.

Table 4. Overview of satellite orbit parameters for satellites visible above the Republic of Serbia.

\begin{tabular}{|l|l|l|l|l|l|l|}
\hline $\begin{array}{l}\text { Parameters } \\
\text { of orbite }\end{array}$ & \multicolumn{6}{|c|}{ Orbit parameter values } \\
\hline $\begin{array}{l}\text { Orbit time } \\
(\mathrm{h})\end{array}$ & $\approx 3$ & $\approx 4$ & $\approx 6$ & $\approx 8$ & $\approx 12$ & $\approx 24$ \\
\hline $\begin{array}{l}\text { Altitude } \\
(\mathrm{km})\end{array}$ & 4163 & 6391 & 10354 & 13982 & 20184 & 35784 \\
\hline Radius $(\mathrm{km})$ & 10541 & 12770 & 16733 & 20720 & 26562 & 42162 \\
\hline $\begin{array}{l}\text { Speed } \\
(\mathrm{km} / \mathrm{s})\end{array}$ & 6149 & 5584 & 4881 & 4434 & 3874 & 3100 \\
\hline
\end{tabular}

\section{VISIBILITY ZONE AND DISTANCE OF SATELLITES}

The zone of visibility of the GSS, ie the area it covers on the Earth, depends on the parameters of the orbit, its position in relation to the ZSS and geographical coordinates, Figure 4. These relations are quite simple if the subsatellite point $P$ is the center of the surface to be covered, while otherwise, they are somewhat more complex. The first similarity of GSS visibility from the Earth's surface can be considered from the geometric relations in Figure 5. When determining the position of GSS towards a ZSS, ie towards MSS or ZSS, the following geometric values are relevant: actual height $h(R+H)$, Earth radius $R$, elevation angle $\varepsilon$, azimuth angle $A$, direct distance $d$, and central angle $\psi$ or subsatellite angle, which corresponds to the angle used to determine the aperture of the satellite dish $\delta$, otherwise known as half the radius of the line of sight. 


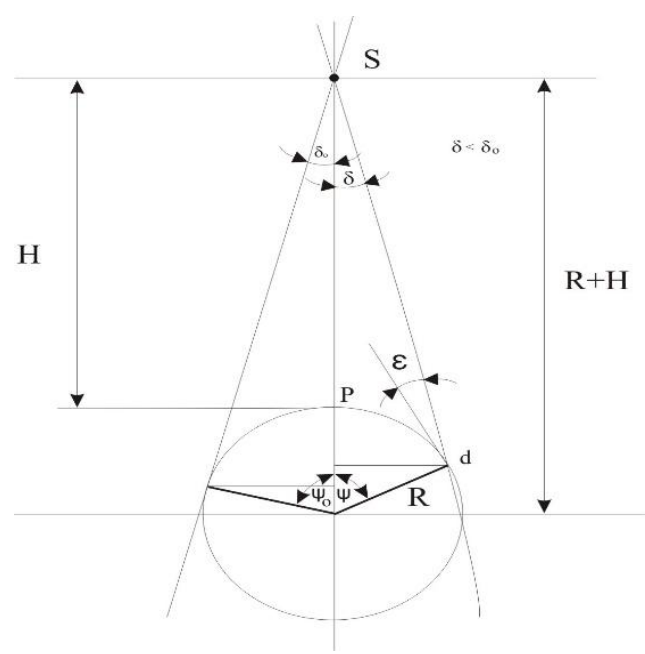

Figure 4. Overview of the visibility zone of a geostationary satellite.

The zone of visibility of the satellite is shown in Figure 4, within a boundary that at all times has the following regular interrelationships:

$$
\delta+\varepsilon+\Psi=90^{0}
$$

Since the height of the orbit $H$ represents the distance of the satellite from the subsatellite point $P$, the expression for the height of the dome is:

$$
H^{\prime}=R(1-\cos \Psi)
$$

The limit of possible coverage with GSS on smooth surfaces of the Earth is reached when the elevation angle is $\varepsilon=0$, ie. when the satellite is on the horizon. This limitation can be expressed by a central angle or angular parameter of the visibility zone, the relation of which is:

$$
\begin{aligned}
& \Psi=\frac{\pi}{2}-\arcsin k=\arccos \frac{R}{R+H}=\arccos \frac{6378,16}{42164,20}= \\
& =\arccos (0.15126956)=81^{0} 17^{\prime} 58.18^{\prime \prime}
\end{aligned}
$$

Therefore, all ZSSs that are located at latitude and longitude above $\psi=81^{\circ}$ will not be covered, because GSS cannot be seen. However, zero elevation angles should be avoided even when maximum coverage is desired because then the noise temperature of the receiving antenna increases. For practical applications, the elevation angle of the receiving antenna must be greater than $5^{\circ}$ (UHF / SVF band) or greater than $20^{\circ}$ (EVF band). Difficult reception also occurs for cases of medium hilly terrain when the satellite can be shaded.

The angle at which a satellite can see the MSS is called the subsatellite angle. The furthest ZSS from the subsatellite point in the corresponding satellite region, ie if the position of the ZSS at the northern and southern latitudes is greater than $\Delta \lambda=70^{\circ}$ or at longitude $\varphi=70^{\circ}$ away east or west of the subsatellite point, the satellite can theoretically see at the minimum elevation angle $\varepsilon=0^{\circ}$. Namely, such a relation can be calculated from simple trigonometric relations (Đukanović 2006b).

An angle $\Psi$ is connected to the angle $\delta$, which can determine the aperture of the radiation beam of the satellite dish. The GSS antenna for global coverage of the Earth is built so that it has a beam of radiation of width $2 \delta^{\prime}=17.3^{\circ}$. According to Figure 5, the following relationship:

$$
\tan \delta=\frac{0.15126956 \cdot \sin \Psi}{(1+0.15126956 \cdot \cos \Psi)}
$$

that is, a $2 \delta^{\prime}$ wide beam aperture provides the maximum coverage possible for a satellite in a synchronous circular orbit.

Determining the position and number of satellites in the visibility zone

The position of the satellite in the zone of visibility of the satellite from the point of signal reception depends on the minimum elevation angle of the receiving antenna, the parameters of the satellite orbit, and the geographical coordinates of the ZSS map. Visibility is also affected by the shape of the orbit (circular or elliptical), (Đukanović 2013).

In order to determine the position of the satellite in relation to the observer from the Earth, Figure 5, ie in relation to the ZSS, specific quantities are used, which are called horizontal coordinates, such as:

1. The elevation angle of the satellite or the angle of the observation point - " $\varepsilon$ ", is formed between the horizon line and the vertical satellite direction seen from the point of view, ie. from the respective position of ZSS.

2. The angle of azimuth - " $A$ " coincides starting from the line of the north-south towards the satellite direction on the horizon seen from the point of view, ie. from the respective position of ZSS.

Otherwise, from point d in Figure 4 or point A in Figure 5, a satellite can be seen at an elevation angle of:

$$
\tan \varepsilon=\frac{\cos \Psi-k}{\sin \Psi}
$$

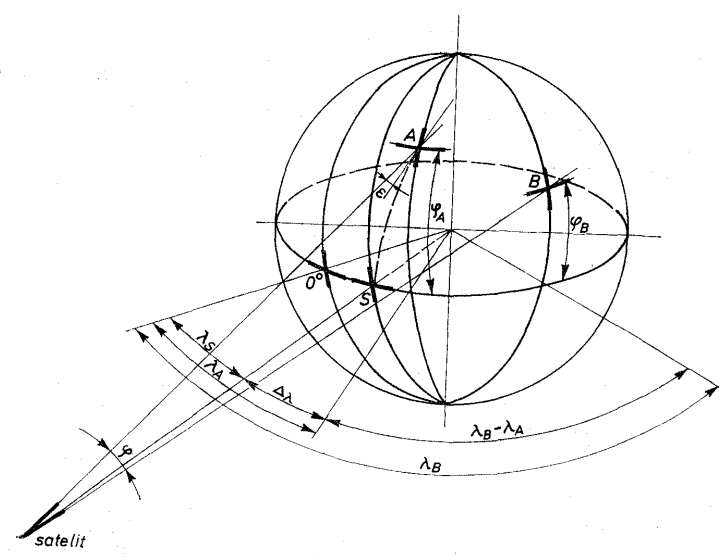

Figure 5. Overview of spatial geometry ZSS-GSS. 
Figure 6 shows a Mercator map with a projection of elevation angles and with one example of that angle for a particular ZSS position. It can be concluded that the ZSS at the respective position ( $\varepsilon=25^{\circ}$ for IOR and $16^{\circ}$ for AOR) has the possibility to alternatively use both GSS to establish a radio connection with any ZSS located within the overlapping area of these two satellites. Satellite azimuth and subsatellite point from the ZSS position, i.e. point A on the earth's surface is obtained from the relation:

$$
\tan A^{\prime}=\frac{\operatorname{tg} \Delta \lambda_{A}}{\sin \varphi_{A}}
$$

However, if the MSS, ie point $A$, is not in the subsatellite point, then the azimuth is obtained from the following relation:

$$
\tan A^{\prime}=\frac{\sin \Delta \lambda_{A}}{\tan \varphi_{A}}
$$

In this case, the magnitude $A^{\prime}$ meridian is the angle between the meridian plane of point $A$ and the plane of the great circle through that and the subsatellite point, while $A$ is the angle between that great circle and the meridian through the subsatellite point. Also in this case the MSS can establish a satellite connection with any ZSS from overlapping satellite areas. Various plans, graphs and tables are used for practical calculation of azimuth angle and GSS elevation for the needs of ZSS, Table 5.

For example, tables for ZSS positions $\left(\varphi^{\prime}\right.$ and $\left.\lambda^{\prime}\right)$ are used to obtain longitudinal differences between ZSS and satellites for four possible ZSS positions: $\mathrm{N}$ and $\mathrm{W}, \mathrm{SIW}, \mathrm{N}$ and $\mathrm{E}$ and $\mathrm{S}$ and E from satellites, as in Figures 6 a, b, cid respectively.

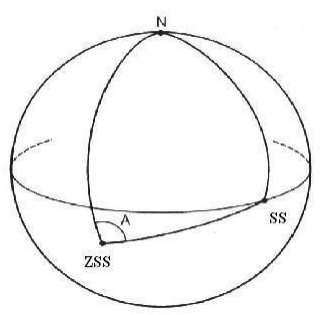

a)

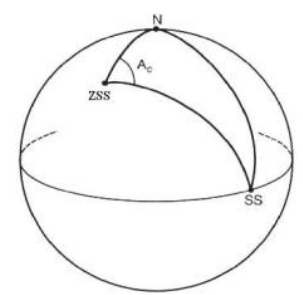

c)

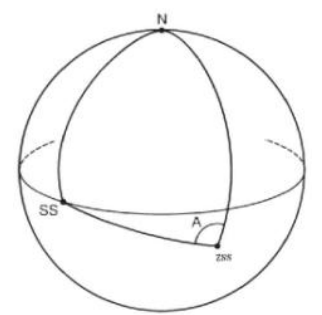

b)

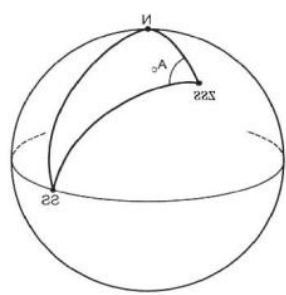

d)
Figure 6. Overview of azimuth angle calculation for all possible ZSS positions.
Table 5. Calculation of the azimuth angle ZSS in relation to the orbital position GSS.

\begin{tabular}{|l|c|}
\hline $\begin{array}{c}\text { The direction of the GSS in } \\
\text { relation to the ZSS }\end{array}$ & $\begin{array}{c}\text { Azimuth angle } \\
\text { calculation }\end{array}$ \\
\hline to $\mathrm{S}$ and W, Fig. 6. a). & $\mathrm{A}=\mathrm{A}^{\prime}$ \\
\hline to S and W, Fig. 6. c). & $\mathrm{A}=180^{\circ}-\mathrm{A}^{\prime}$ \\
\hline to S and W, Fig. 6. d). & $\mathrm{A}=180^{\circ}+\mathrm{A}^{\prime}$ \\
\hline to $\mathrm{S}$ and W, Fig. 6. b). & $\mathrm{A}=360^{\circ}$ - $\mathrm{A}^{\prime}$ \\
\hline
\end{tabular}

If the ZSS is at a higher altitude then the elevation angle is compensated by the following magnitude:

$$
x=\arccos \left(1-\frac{N}{R}\right)
$$

where $\mathrm{N}$ is the altitude of the observer. For example, if the ZSS is located at an altitude of $1000 \mathrm{~m}$, it is $\mathrm{x} \approx 1^{\circ}$.

Based on the previous analysis, the following can be concluded: If an antenna with a minimum elevation angle $\left(5^{\circ}\right)$ is used at the receiving station, then those GSS whose longitudinal position in orbit is in the range of $50^{\circ} \mathrm{W} \mathrm{W}$ (west) can be seen from the territory of Serbia. to $90^{\circ} \mathrm{E}$ (east),

\section{CONCLUSION}

In this paper, we deal with the general technical, spatial, and temporal characteristics of satellite telecommunications systems. Particular attention was paid to the peculiarities of the territory of the Republic of Serbia in terms of implementation and use of modern satellite telecommunication infrastructure. The use of various satellite telecommunication systems lead to configure and exploit next-generation networks, especially modern communications such as $5 \mathrm{G}$ technology and IoT. Their work cannot be imagined without the high speeds and high frequencies that allow us to transmit a wealth of information from short messages/news to HD video on a mobile phone. The paper presents data that give a numerical and graphical overview of geostationary satellites visible from the territory of the city of Belgrade, depending on the orbital position and the associated angle of azimuth and elevation, which would also be valid for the leading territory of Serbia.

\section{ACKNOWLEGMENTS}

This paper was supported by the Ministry of Education, Science and Technological Development of the Republic of Serbia, and these results are parts of the Grant No. 451-0368/2020-14/200132 with University of Kragujevac - Faculty of Technical Sciences Čačak. 


\section{REFERENCES}

Andrews, L. C., \& Phillips, R. L. 1998. Laser Beam Propagation Through Random Media, SPIE.

David Darling 2020. https://daviddarling.info/encyclopedia/ M/Molniya-type_orbit.html

Đukanović, S. 2004. Izviđanje satelitskih komunikacija u funkciji savremenih operacija 1,Vojnotehnički glasnik. 52(34), pp. 390-399.

Đukanović, S. 2006a. Elektronsko izviđanje komercijalnih satelitskih komunikacija, Bezbednost, Beograd - Info, 48(1), pp. $100-117$.
Đukanović, S. 2006b. Neke specifičnosti izviđanja satelitskih veza u L opsegu, TELFOR.

Đukanović, S. Papić, V., \& Đurović, Ž. 2013, Procena učestanosti kanala u satelitskim komunikacijama korišćenjem tehnika robusnog filtriranja I skrivenih Markovljevih modela, ETRAN.

Gligorijević, M., \& Đukanović, S. 2011, GPS - Global positioning system and its applications in policing, Archibald Reiss Days.

Panić, S., Stefanović, M., Anastasov, J., \& Spalević, P. 2013. Fading and Interference Mitigation in Wireless Communications, CRC Press.

Wolfram, http://functions.wolfram.com/ 